\title{
Characterization and hemocompatibility assessment of porous composite scaffolds with a biomimetic human clavicle macrostructure
}

\author{
Claudio Jesus Chi Perera ${ }^{1}$. Melissa Guadalupe Castillo Baas ${ }^{2}$ (D) Gabriela Abigail Alcocer Lara ${ }^{2}$. \\ Shirley Isaai Ramos Borges ${ }^{3}$ • Alma Leticia Rodríguez Guzmán ${ }^{2}$ • Irving Fernández Cervantes ${ }^{4}$. \\ Nayeli Rodríguez Fuentes ${ }^{5}$
}

Received: 20 January 2019 / Accepted: 22 August 2019 / Published online: 29 August 2019

(C) The Author(s) 2019

\begin{abstract}
One objective of tissue engineering is to support tissue regeneration using biomaterials that replace the damaged area, such as polylactic acid (PLA), sodium alginate (SA), and hydroxyapatite (HA). Therefore, determining the hemocompatibility of composite structures made from these materials is vital to rule out the possible blood trauma that such structures may cause. This work evaluates the percentage of hemolysis in porous PLA-SA-HA scaffolds with clavicle morphology made with different printing parameters $(200,400,600$, and 800 Voronoi tessellations) using additive manufacturing technology. These materials were characterized with infrared Fourier transform spectroscopy (FTIR), X-ray diffraction (XRD), and scanning electron microscopy (SEM-EDX) techniques. The FTIR analysis showed the main functional groups of the materials that make up the composite material, i.e. hydroxyapatite. The SEM micrographs showed a topology that mimics the trabecular sections of human bone tissue. The presence of the hydroxyapatite phase was confirmed by XRD. The hemolysis test was significant $(p<0.0126)$ and indicated that the material of 200 tessellations had a lower percentage of hemolysis (3.29\%), if compared to the other materials $(p<0.05)$, thus suggesting that said composite has a greater potential for tissue engineering applications of bone regeneration.
\end{abstract}

Keywords Bone $\cdot$ Compatibility $\cdot$ Hemolysis $\cdot$ Scaffold

Melissa Guadalupe Castillo Baas melissa.castillo.baas@gmail.com

1 Escuela de Ingeniería, Universidad Modelo, Carretera a Cholul 176, 97305 Mérida, Yucatán, Mexico

2 Facultad de Medicina, Universidad Autónoma de Yucatán, Avenida Itzáes No. 498 x 59 y 59 A, Centro, 97000 Mérida, Yucatán, Mexico

3 Facultad de Química, Universidad Autónoma de Yucatán, Calle 43 613 x Calle 90, Colonia Inalámbrica, 97069 Mérida, Yucatán, Mexico

4 Universidad Aeronáutica en Querétaro, Carretera Estatal 200 Querétaro-Tequisquiapan No. 22154 76270. Colón, Querétaro, Mexico

5 Laboratorio de Biomateriales, Consejo Nacional de Ciencia y Tecnología, Calle 43 No. 130 x 32 y 34, Chuburná de Hidalgo, 97205 Mérida, Yucatán, Mexico

\section{Introduction}

Currently, bone regeneration is an important area of study in the field of tissue engineering. Bones fractures can threaten patient life quality: e.g. the fracture of the middle third segment of the clavicle, being the most frequent upper limb fracture in minors [1]. Biomaterials can be used to heal clavical fractures by replacing the damaged area, thus coming into contact with living tissues and/or biological fluids, repairing the lesion, or promoting tissue regeneration. Similarly, biomaterials can be bioinert or bioactive (promote cellular activity).

The three most common biomaterial include hydroxyapatite (HA), polylactic acid (PLA), and sodium alginate (SA). $\mathrm{HA}$ is a biocompatible ceramic that provides active sites for biomineralization and cell adhesion through osteoconduction (osteoblast growth), osteoinduction (ontogenic differentiation capacity), and osteointegration (generation of extracellular matrix). Unfortunately, HA has low mechanical properties; that is, it is fragile. On the other hand, PLA is a polyester 
obtained from renewable resources such as starch, and it has good mechanical properties; however, PLA rarely has desirable bioactive potential [2]. Finally, SA is an antonic polyelectrolyte used as a gelling agent. Similarly, it can serve as a means for cellular transport and tissue regeneration [3].

When biomaterials such as PLA, HA, and SA are combined, composite material are created. These materials are formed by a matrix (commonly made of polymeric materials such as PLA) and a load (made of ceramic materials). Likewise, composite materials widely very in morphology, length-scale, and superior properties. As a result, their study is of increasing interest [4].

Commonly, composites are made through Extrusion Deposition Additive manufacturing (EDAM), a form of 3D printing. This process has proven to be an efficient, low-cost, and accessible alternative for generating polymeric matrices [5]. Similarly, to create composites, the matrix surface is modified using colloids generated from polymers (e.g. colloid generated using SA-HA) that get in the presence of bioactive ceramic materials [6].

Hemocompatibility testes are used to determine whether certain health products of biomaterials can cause thrombosis, coagulation, platelet activation, activation of the complement system, and erythrocyte alterations (hemolysis tests). Likewise, these tests can help determinate material's viability for tissues engineering applications. The goal of this work is in characterize the composition and structure of PLA-SA-HA composite scaffolds made with different printing parameters that mimic the macro and microstructure of the clavicle. Similarly, we seek to evaluate hemolysis in these scaffolds to improve their biocompatibility and bioactivity.

\section{Methodology}

\subsection{Hydroxyapatite extraction from bovine bone}

Hydroxyapatite was extracted from fresh bovine bones (metatarsus), which were subjected to cleaning and disinfection processes by means of pressure cooking at $120{ }^{\circ} \mathrm{C}$ for five hours to deproteinize the bones. Subsequently, was calcined at $850{ }^{\circ} \mathrm{C}$ for one hour and immediately at $900{ }^{\circ} \mathrm{C}$ for two hours. Once cooled, the HA powder was obtained by grinding. In the end, $120 \mathrm{~g}$ of hydroxyapatite powder was obtained from $300 \mathrm{~g}$ of bovine bone.

\subsection{Computational modeling of the scaffold}

We design porous composite scaffolds was done by mimicking the macro structure of the human clavicle and providing the porosity by dividing the structure into small fractions with fractal morphology, as shown in Fig. 1. The simulation of the model was performed using software AUTODESK ${ }^{\mathrm{TM}} 3 \mathrm{DS}$ MAX $^{\mathrm{TM}}$, V.2015. Several pore size configurations were tested. To change pore size configuration, we changed the number of tessellations between 200, 400, 600 and 800 and the Shell between $0.1 \mathrm{~cm}$ to $1 \mathrm{~cm}$.

\subsection{Scaffolding by means of 3D printing}

To print the scaffold, we used a MakerBot printer with a $0.2 \mathrm{~mm}$ nozzle and a $1.75 \mathrm{~mm}$ diameter PLA filament. Similarly, we used the printer's software program to generate the polymeric matrix.
Fig. 1 Computational models generated in 3DS MAX.

Simulated models with A) 200 tessellations, B) 400 tessellations, C) 600 tessellations and D) 800 tessellations

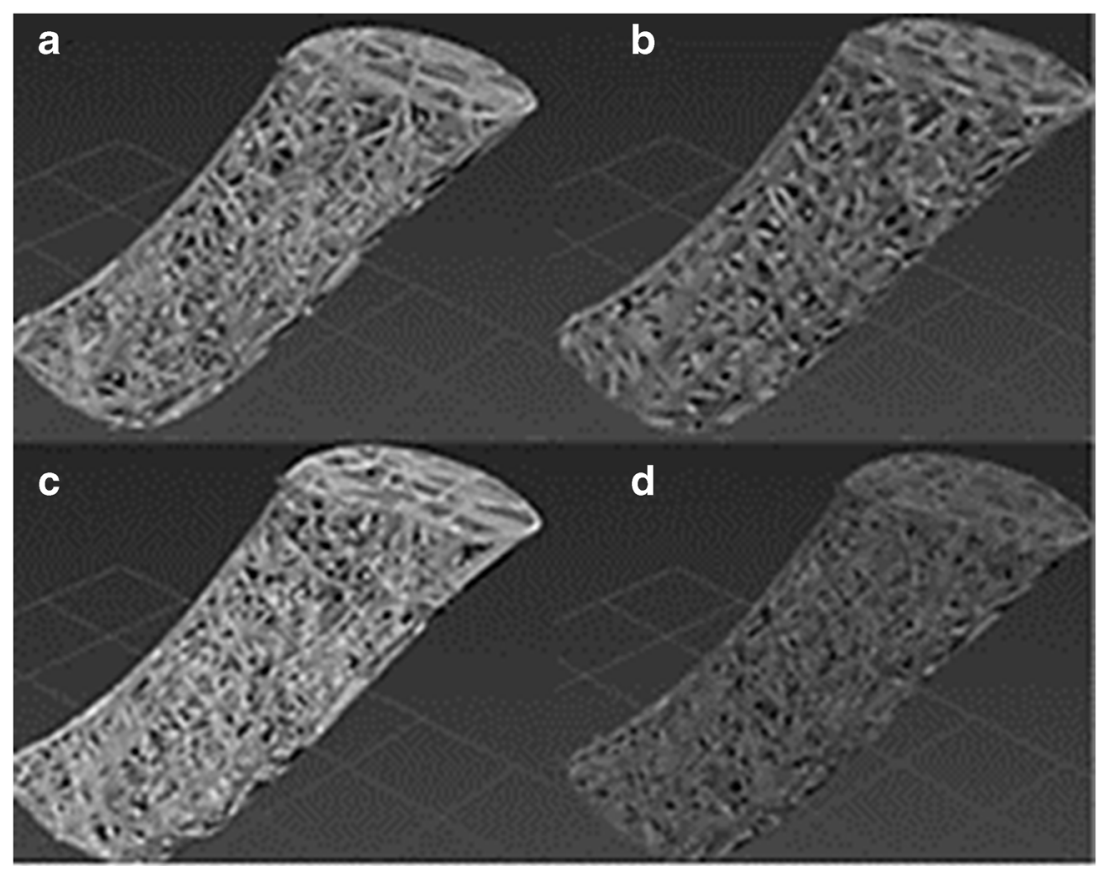




\subsection{Surface modification}

To generate of the composite material (PLA-SA-HA), a suspension of SA-HA was generated by sonicating HA and SA in water and mixing in a 1:1 ratio. The PLA polymer matrix was submerged in the SA-HA suspension in a batch reactor with continuous agitation, for $15 \mathrm{~min}$. Finally, the scaffolds were dried at $70{ }^{\circ} \mathrm{C}$ for $25 \mathrm{~min}$.

\subsection{Characterization of the scaffolding}

X-ray diffraction (XRD) We employed An X-ray diffractor Bruker D8-Discover X-ray light beam for powders. The Xray diffractometer used $\mathrm{Cu} \mathrm{K}$ radiation (wavelength = $0.15418 \mathrm{~nm}$ ) to determine the crystalline structure of the obtained hydroxyapatite phase.

Scanning electron microscopy (SEM-EDX) We analyzed the morphology using the electron scanning microscope (SEM) JSM-6610LV from Jeol.

Infrared Fourier transform spectroscopy (FTIR) We used a FTIR infrared spectrophotometer with ATR, VERTEX model 70 , to determine functional groups and confirm the presence of HA.

\subsection{Hemolysis}

We obtained peripheral blood from a healthy volunteer and collected in BD Vacutainer tubes with EDTA, according to ISO 10993-4:2017 and the Escuderos-Castellanos methodology [7].

The four scaffolds of $17 \mathrm{~g}$ each, were sterilized by UV light. Subsequently, they were placed in contact with $5 \%$ of human erythrocytes and incubated at $37{ }^{\circ} \mathrm{C}$ for one hour. The sample absorbance was measured at $415 \mathrm{~nm}$ in a spectrophotometer (Cytation 3). The hemolysis percentage was calculated according to the following equation:

$\%$ Hemolysis $=\left(\frac{(\text { OD test }- \text { OD negative })}{(\text { OD positive }- \text { OD negative }}\right) 100$

Where OD test corresponds to the optical density of each tested scaffold, OD negative refers to the negative control ( $500 \mu \mathrm{l}$ of erythrocytes washed with $0.9 \%$ saline solution), and OD positive stands for to the positive control $(500 \mu \mathrm{l}$ of washed erythrocytes and $500 \mu \mathrm{l}$ of distilled water). The differences between treatments were analyzed with a one-way ANOVA test for repeated samples $(p<0.05)$ and the Tukey multiple comparison post-hoc test $(p<0.05)$ using the GraphPad Prism 5 software.

\section{Results}

\subsection{X-ray diffraction (XRD)}

Figure 2 shows the diffraction pattern of the composite material [section $\mathrm{A})]$, the trabecular bone tissue [section $\mathrm{B})]$, and the HA used to generate the composite scaffolds [section C)]. In the three patterns, the peaks (002), (210) and, (211) with angles of $22.89^{\circ}, 25.99^{\circ}$ and $31.89^{\circ}$ respectively, which are found in the JCPDS crystallographic sheet: 09-432, representing the crystallographic planes characteristic of the structure of calcium phosphate in the hydroxyapatite phase.

Such results confirm the presence of the crystalline phase of calcium phosphate in the composite material, which is also present in human bone tissue, thus maintaining similarity in the pattern [8].

Such results confirm the presence of the crystalline phase of calcium phosphate in the composite material, wich is also present in human bone tissue, thus maintaining similarity in the pattern [8]. The crystal size of the HA, $t$ (hkl), was calculated from the XRD pattern using the Debye-Scherrer equation [10] as follows:

$t(h k l)=\left(\frac{0.9 \lambda}{B \cos (\theta(h k l)}\right)$

where $\lambda$ is the wavelength of the incident beam of the monochromatic radiation, $\mathrm{B}$ is the average maximum peak width (FWHM) at the maximum intensity, $\theta$ (hkl) is the diffraction angle that satisfies Bragg's law for plane (hkl) is the size. We used the peak from plane (002) of the XDR pattern to calculate the crystal size of HA, as reported in the literature $[9,10]$. According to eq. 1 (Debye- Scherrer equation), the crystal has an average size of $8.31 \mathrm{~nm}$. As reported in the literature,

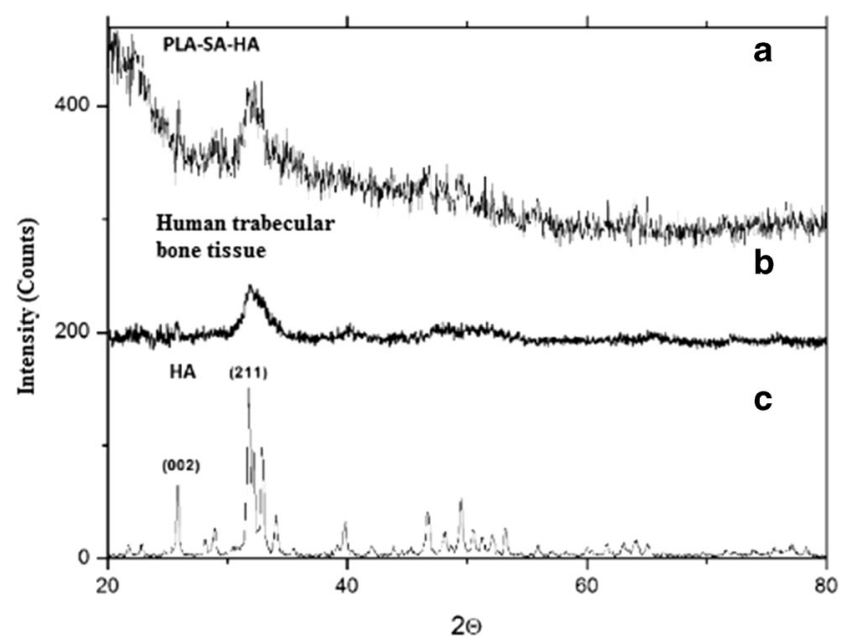

Fig. 2 Particulate diffraction pattern: A) composite material PLA-SAHA, B) human trabecular bone and C) HA obtained 
a crystal size at the nanometric level is desirable to apply HA in bone tissue engineering $[9,10]$.

\subsection{Scanning electron microscopy (SEM-EDX)}

Figure 3 shows the electron micrographs for the ceramic material HA [section A) -D)], and the composite material [section E) and F)]. The ceramic material showed particle agglomerates with an irregular spherical morphology and sizes larger than $10 \mathrm{~m}$. A similar morphology was noticed in the composite material. Also, we found that the larger the agglomerates, the smaller the particles.

This configuration may be due to the fact that these microparticles can be composed of aggregates of smaller particles (even in the order of nanometers), as a result of to the selforganization phenomenon occurring in materials when there is a break of symmetry allowing the nucleation process to occur, and leading to the formation of larger structures [10]. This phenomenon would explain why HA has a theoretical crystal size of approximately $8 \mathrm{~nm}$ as measured by $\mathrm{X}$-ray diffraction. However, to corroborate this phenomenon it is necessary to perform tests through atomic force microscopy (AFM) or transmission electron microscopy (TEM).

\subsection{Infrared Fourier transform spectroscopy (FTIR)}

Figure 4 shows the FTIR spectrum of the extracted HA with the absorption bands characteristic of HA. Absorption bands at $1085,1028,961,858 \mathrm{~cm}-1$ correspond to the vibrations of phosphate groups PO4 in HA. The first band at $1085 \mathrm{~cm}-1$ results from a triple vibration of degenerate asymmetric stretching. The vibration of the PO bond in the phosphate group appears at $1028 \mathrm{~cm}-1$. The peak at $961 \mathrm{~cm}-1$ to the non-degenerate symmetric stretching of the PO bond and the
Fig. 3 Electron micrographs of the HA ceramic material: a) with a magnification of: a) $150 x$, b) $300 x$, c) 2300x and d) 1500x and of the PLA-SA-HA composite material with a magnification of $1300 \mathrm{x}$ and $130 \mathrm{x}$

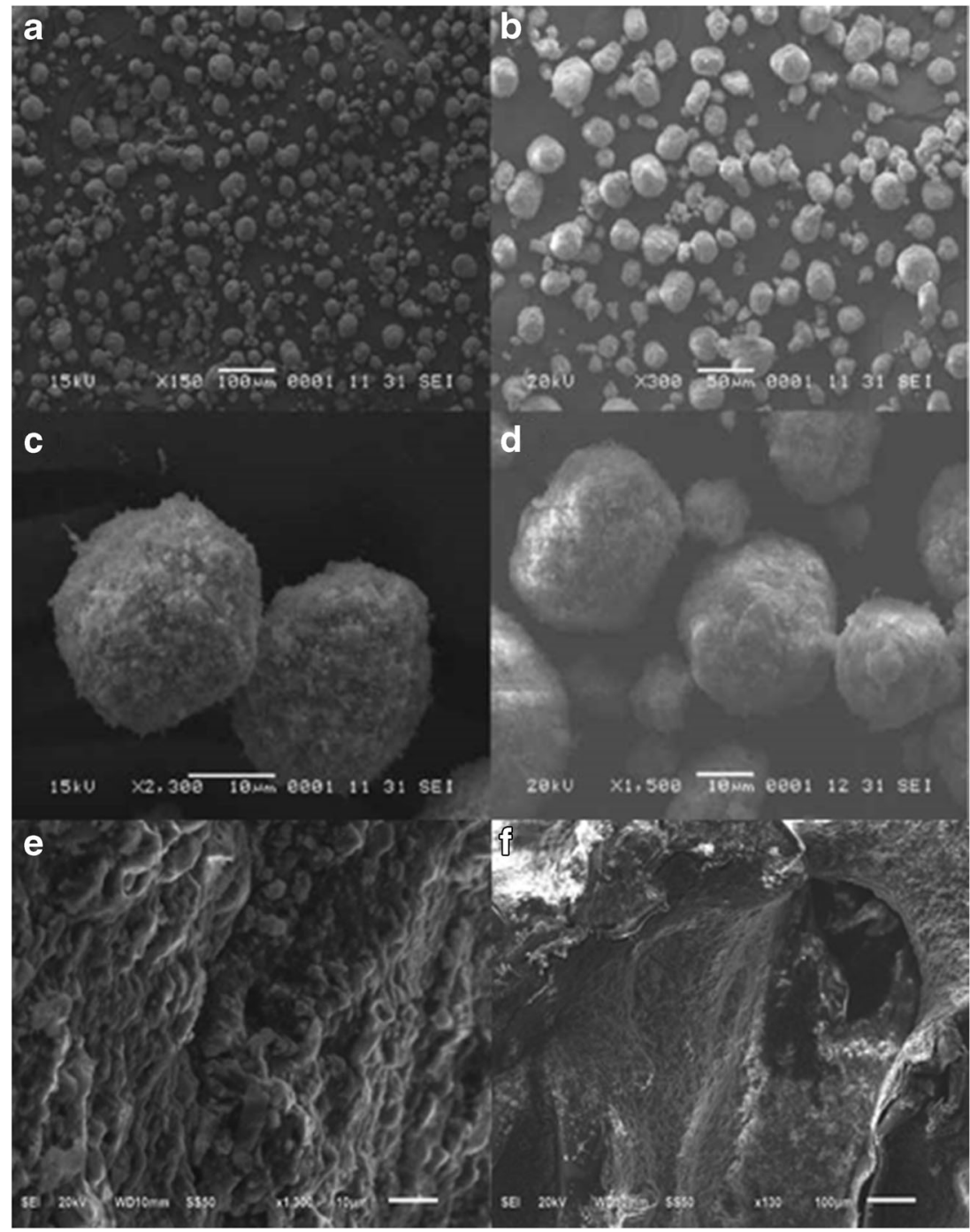




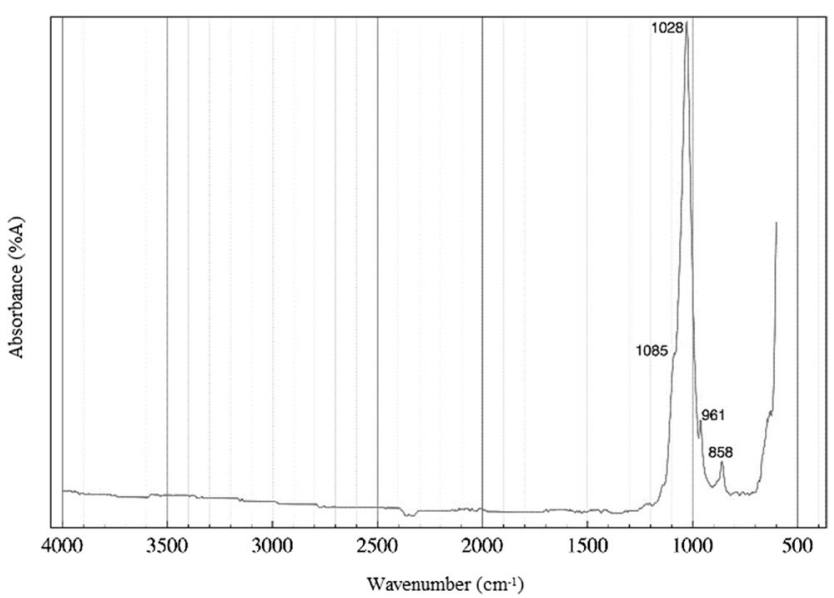

Fig. 4 Fourier transform infrared spectrum of the extracted HA

bands in the fingerprint region at $858 \mathrm{~cm}-1$ correspond to vibrations of triple degenerate flexion of the OPO link. It is important to mention that the hydroxyapatite extracted in this work is of significant biological interest, since it presents a $\mathrm{Ca} /$ $P$ ratio of 1.4 wich is closer to the ratio found in bone tissue of 1.5 , as compared stoichiometrically [11].

\subsection{Hemolysis}

Finally, Fig. 5 shows hemolysis percentages after contact with the scaffolds made with different printing conditions. Only the 200 tessellations condition resulted in less than $5 \%$ of hemolysis. The ANOVA test for hemolysis was significant with a $p$ value of $<0.0126$. As for the results of the post-hoc Tukey test, we found differences between conditions with a $\mathrm{p}$ value of $<0.05$.

\section{Discussion}

In this work, we tested different numbers of tessellations to print the PLA using the VoroFrag plugin, which dictates scaffold pore size. By increasing the number of tessellations, the internal structure of the model became more intricate with

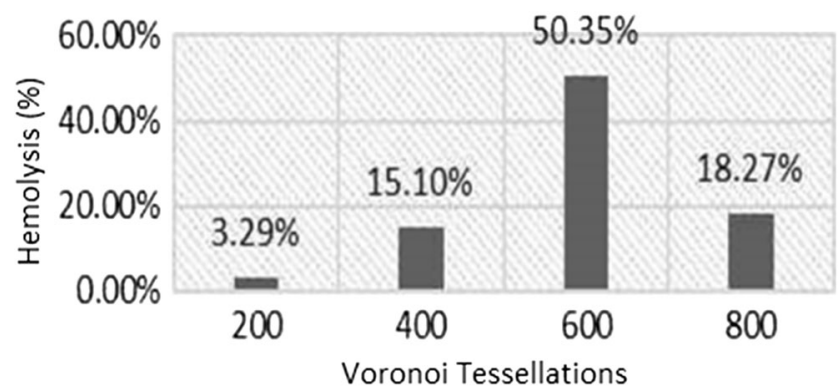

Fig. 5 Graph of the hemolysis percentage according to the different printing conditions smaller pore size, thus generating a larger number of simulated trabeculae and elongations within our scaffolding. This characteristic is particularly, since it highly resembles the natural structure of the human bone, and together with the intrinsic properties of the composite material, help with the generation of bone matrix. This is demonstrated by the results obtained with the FTIR, DRX, and SEM-EDX techniques. Experts claim that hemolysis percentages below 5\% for a material are highly hemocompatible $[10,11]$, which is the case in our 200 tessellations model. Considering that an erythrocyte is $7.8 \mathrm{~nm}$ by $2.6 \mathrm{~nm}$ in size, the pore size of the scaffold should be adequate to prevent the accumulation of these cells and cause some damage to human tissue. On the other hand, an increase in hemolysis can be caused by an intervention of calcium phosphate on the erythrocyte's cellular structure, thus allowing the passage of the SA-HA gel to the scaffold pores and coming into contact with the membrane. This ultimately causes erythrocyte programmed cell death [12].

\section{Conclusions}

This research demonstrates that obtaining HA from bovine bone is a viable, low-cost, and fast alternative for generating composite scaffolds with bioactive properties. Furthermore, we managed to generate a model that mimics the intrinsic properties of the human clavicle. This model was made from a composite material based on PLA, SA, and HA. In conclusion, we found an inverse relationship between pore size and hemocompatibility in PLA-SA-HA composite scaffolds. The 200 tessellations model (i.e. greater porosity) showed $3.29 \%$ of hemolysis and has the potential to act as a biocompatible scaffold. Therefore, as further research, we suggest performing additional hemocompatibility tests (e.g. platelet aggregation and morphology and viability of mononuclear cells).

Acknowledgements We express our gratitude to the Centro de Investigación Científico de Yucatán (CICY) for their valuable help with heat treatment and hemolysis tests. We also thank the Centro Universitario de Vinculación BUAP, Dr. Marco Antonio Morales Sánchez, Efraín Rubio Rosas, and Ricardo Agustín Serrano for their support in the characterizations. Universidad Modelo and Universidad Autónoma de Yucatán for providing us the facilities conduct this project.

\section{Compliance with ethical standards}

Conflict of interest The authors declare no potential conflicts of interest in relation to this research paper.

Ethical approval This article does not contain any studies with human participants or animals performed by any of the authors.

Informed consent Informed consent was obtained from all individual participants included in the study. 
Open Access This article is licensed under a Creative Commons Attribution 4.0 International License, which permits use, sharing, adaptation, distribution and reproduction in any medium or format, as long as you give appropriate credit to the original author(s) and the source, provide a link to the Creative Commons licence, and indicate if changes were made. The images or other third party material in this article are included in the article's Creative Commons licence, unless indicated otherwise in a credit line to the material. If material is not included in the article's Creative Commons licence and your intended use is not permitted by statutory regulation or exceeds the permitted use, you will need to obtain permission directly from the copyright holder. To view a copy of this licence, visit http://creativecommons.org/licenses/by/4.0/.

\section{References}

1. Egol K, Koval K, Zuckerman J. Handbook of Fractures. 5th ed. Wolters Kluwer; 2015.

2. Altamirano AA, Vargas N, Vázquez FC, Vargas T, Montesinos JJ, Alfaro E, et al. Biocompatibilidad de andamios nanofibrilares con diferentes concentraciones de PLA/Hidroxiapatita. Odovtos-Int J Dent Sc. 2016;18:3. https://doi.org/10.15517/ijds.v0i0.25987.

3. Herrera AP, Acevedo MT, Castro MI, Marrugo LJ. Preparación de nanopartículas de quitosano modificadas con alginato de sodio con potencial para la liberación controlada de medicamentos. Revista EIA. 2016;12(3):E75-83. https://doi.org/10.14508/reia.2016.12. e3.75-83.

4. Askeland D, Wright W. Science and engineering of materials. 3rd ed. Boston: Cengage Learning; 1998.
5. Canessa E, Fonda C, Zennaro M. Low-cost 3D Printing for Science, Education and Sustainable Development. The Abdus Salam International Centre for Theoretical Physics (ICTP) 2013.

6. Escudero-Castellanos A, Ocampo-García B, Domínguez-García M, Flores-Estrada J, Flores-Merino M. Hydrogels based on poly (ethylene glycol) as scaffolds for tissue engineering application: biocompatibility assessment and effect of the sterilization process. J Mater Sci: Mater Med. 2016;27(176):1-10. https://doi.org/10. 1007/s10856-016-5793-3.

7. Ghista DN. Biomedical Science, Engineering and Technology. 2012.

8. Yu X, Tang X, Gohil SV, Laurencin CT. Biomaterials for Bone Regenerative Engineering. Adv Healthc Mater. 2015;4(9):1268-85.

9. Morales MA, Fernández-Cervantes I, Agustín-Serrano R, Anzo A, Sampedro MP. Patterns formation in ferrofluids and solid dissolutions using stochastic models with dissipative dynamics. EUR. Phys. J. B. 2016;89:182. https://doi.org/10.1140/epjb/ e2016-70344-7.

10. Bett J, Christner L. Studies of the Hydrogen Held by Solids. XII. Hydroxyapatite Catalysts. J Am Chem Soc. 1967;77:5535-41.

11. Pal K, Banthia A, Majumdar D. Biomedical evaluation of polyvinyl alcohol-gelatin esterified hydrogel for wound dressing. J Mater Sci: Mater Med. 2007;18:1889-94. https://doi.org/10.1007/s10856007-3061.

12. Manzur F, Álvarez N, Moneriz C, Corrales H, Cantillo K. Eriptosis: mecanismos moleculares y su implicación en la enfermedad aterotrombótica. Rev Colomb Cardiol. 2016;23(3):218-26. https://doi.org/10.1016/j.rccar.2015.09.004.

Publisher's note Springer Nature remains neutral with regard to jurisdictional claims in published maps and institutional affiliations. 